\title{
Fusing time, frequency and shape-related information: Introduction to the Discrete Shapelet Transform's second generation (DST-II)
}

\section{Rodrigo Capobianco Guido}

Instituto de Biociências, Letras e Ciências Exatas, Unesp - Univ Estadual Paulista (São Paulo State University), Rua Cristóvão Colombo 2265, Jd Nazareth, 15054-000, São José do Rio Preto - SP, Brazil

\section{A R T I C L E I N F O}

\section{Article history:}

Received 21 March 2017

Revised 16 July 2017

Accepted 27 July 2017

Available online 29 July 2017

\section{Keywords:}

Information fusion

Discrete shapelet transform (DST)

Time-frequency-shape (TFS) joint analysis

Discrete wavelet transform (DWT)

Pattern analysis

Signal processing

\begin{abstract}
A B S T R A C T
This article introduces the second generation of the Discrete Shapelet Transform (DST-II), a tool created for fusing three types of information: time, frequency and shape-related. Considered a particular Discrete Wavelet Transform (DWT), it allows a productive time-frequency-shape (TFS) joint analysis. In the proposed approach, both the procedure to attain the corresponding filters coefficients and the interpretation of the transformed signal are simplified in relation to the usage of its predecessor, i.e., the DST. Throughout the article, the DST-II formulation is described in detail, including a numerical example, a prototype for use in a diversity of fields and an application on spike and overlap sorting, reassuring the efficacy of the new transform.
\end{abstract}

(c) 2017 Elsevier B.V. All rights reserved.

\section{Introduction}

Since Alfréd Haar, the father of wavelets, introduced fundamental concepts in 1909 [1] and Ingrid Daubechies, the mother of wavelets, brightly consolidated them decades later [2], the Discrete Wavelet Transform (DWT) [3] has been placed at the forefront of signal analysis, fusing two types of information: temporal and spectral. Through the years, many other scientists have published their relevant contributions to the field of wavelet theory. Palghat Vaidyanathan, Martin Vetterli, Stéphane Mallat, David Donoho, Gilbert Strang and others are examples of unforgettable names, just to mention a few. Additionally, our information fusion community has continuously presented DWT-based and DWTinspired advances, as shown in [4-13].

In order to further evolve ordinary wavelet analysis, the Discrete Shapelet Transform (DST) was recently created and presented to the scientific community, as documented in [14], in a previous cooperative work of mine. Exactly as the Discrete Wavelet Transform (DWT) does [15], the DST allows the time-support of frequencies to be found, however, with a special advantage: concomitantly, it quantifies the degree of similarity between the signal under analysis and a pre-specified shape. Its work principle consists of a fractal-based criterion [16] used to redefine the original
Daubechies' DWTs in such a way that a time-frequency-shape (TFS) joint analysis is performed. Thus, the DST fuses three types of information: temporal, spectral and shape-related.

On one hand, the original DST formulation demonstrated that TFS joint analysis is feasible. On the other, my objective this time is to improve that technique by defining the second generation of the transform, i.e., DST-II. Particularly, the new tool replaces the fractal-based criterion used for shape matching by a correlationbased formulation, favouring the solution of the non-linear system of equations that produces the filters coefficients and allowing a simplified interpretation of the transformed signal. Thus, the DSTII is better than its predecessor for joint TFS analysis, stimulating its usage in a diversity of fields.

In suggesting possible future trends for the scientific community, this paper is organised as follows. Supported by a short review on DSTs and the original Daubechies' DWT, presented in Section 2, Section 3 introduces the DST-II and its inverse (IDST-II). Proceeding, Section 4 shows a numerical example, while Section 5 describes the tests and results obtained during the analysis of simulated and biological data and, lastly, Section 6 reports the conclusions that are followed by the references. Readers of this article are strongly encouraged to learn my previous piece on the original DST [14] before proceeding any further.

E-mail address: guido@ieee.org

URL: http://www.sjrp.unesp.br/ guido/ 


\section{A short review}

\subsection{DSTs: the first generation}

A deep review on DSTs is superfluous due to the detailed description presented previously in [14]. However, there are a few important points to be mentioned regarding the elements associated with that transform. Similarly to the ordinary DWT, they are:

- $p[\cdot]$ and $q[\cdot]$, so that $q_{k}=(-1)^{k} p_{N-k-1}$, form the quadrature mirror filter (QMF) [3] pair of finite impulse response (FIR) filters [17] with support-size $N \geq 4$ used for signal analysis, being $N$ even. They present, respectively, low-pass and high-pass frequency responses with not necessarily linear phases [17]. Accordingly, these are the filters used in conjunction with Mallat's algorithm [18] to obtain the transformed signal from the input, exactly as in the original DWTs for which they are usually known as $h[\cdot]$ and $g[\cdot]$;

- $\bar{p}[\cdot]$, so that $\bar{p}_{k}=p_{N-k-1}$, and $\bar{q}[\cdot]$, so that $\bar{q}_{k}=(-1)^{k+1} p_{k}$, form the pair of filters used for signal re-synthesis. In the scope of the DWT, they would be respectively known as $\bar{h}[\cdot]$ and $\bar{g}[\cdot]$;

- $\Gamma(x)=\sum_{k} p_{k} \Gamma(2 N-k)$ and $\Theta(x)=\sum_{k} q_{k} \Gamma(2 N-k)$, respectively known as major shapelet and minor shapelet, correspond to scaling and wavelet functions of the DWT [3], i.e., $\Phi(x)$ and $\Psi(x)$;

- the conditions $\bar{P}[z]=Q[-z], \quad \bar{Q}[z]=-P[-z]$ and $\bar{P}[z] P[z]+$ $\bar{Q}[z] Q[z]=2 z^{-N+1}$, all in $Z$ domain [17], imply that $p[\cdot], q[\cdot], \bar{p}[\cdot]$ and $\bar{q}[\cdot]$ form a perfect-reconstruction filter bank (PRFB) [3].

Particularly, the procedure to obtain the DST filter $q[\cdot]$ is the same used to generate the Daubechies' filter $g[\cdot]$, as reviewed ahead, albeit with one difference: the former formulation replaces one vanishing moment condition from the latter by a fractal-based matching equation. The DST-II, however, is based on a different approach.

Complementarily, it is important to recall that the $\operatorname{DST}(s[\cdot])$ preserves the length of the input signal $s[\cdot]$, hereafter referred to as $X$, that is a power of 2 . Furthermore, $X$ allows for the decomposition until level $j=\left(\frac{\log (X)}{\log (2)}\right)$. Once $s[\cdot]$ is decomposed, two other signals with lengths $\frac{X}{2}$ are produced: master and second-rated. The former and the latter result, respectively, from the convolution of $s[\cdot]$ with $p[\cdot]$ and the convolution of $s[\cdot]$ with $q[\cdot]$, both followed by a downsampling by 2 and a wrap-around procedure [18]. Lastly, the concatenation of master with second-rated characterizes the DST. From the former, the decomposition can continue recursively until reaching the highest possible level. DST-II inherits all the terminology and decomposition procedures from DST.

\subsection{The discrete Daubechies' transform}

There are distinct ways to explain how the Daubechies's wavelets [3] were constructed. Particularly, that of my current interest, which first produces the high-pass filter, i.e., $g[\cdot]$ with support-size $N$, and then generates the other elements, i.e., $h[\cdot]$, $\bar{h}[\cdot], \bar{g}[\cdot], \Phi(x)$ and $\Psi(x)$, based on it, will be reviewed here. The specific procedure is:

- STEP Daub ${ }_{1}$ : Force $g[\cdot]$ to have unitary energy so that the DWT preserves that of the input signal, i.e.,

$$
\sum_{k=0}^{N-1} g_{k}^{2}=1 .
$$

This condition is equivalent to others, as $\sum_{k=0}^{N-1} h_{k}=\sqrt{2}$, implying that the scaling function has one non-vanishing moment;
- STEP Daub 2 : Impose $\frac{N}{2}$ vanishing moments on the wavelet function, i.e.,

$\sum_{k=0}^{N-1} g_{k} k^{b}=0 \quad$,

for $b=0,1, \ldots, \frac{N}{2}-1$;

- STEPDaub D $_{3}$ : Define $\frac{N}{2}-1$ orthogonality conditions related to the translations of the filter so that the transformation matrix used to carry out Mallat's algorithm [18] is orthogonal, allowing signal re-synthesis based on its transpose:

$\sum_{k=0}^{N-1} g_{k} g_{k+2 l}=\delta_{0, l}$,

being $\delta$ the Dirac delta and $l \in \mathcal{Z}$;

- STEP Daub ${ }_{4}$ : Group together the only equation of step Daub ${ }_{1}$, the $\frac{N}{2}$ equations of step Daub $_{2}$ and the $\frac{N}{2}-1$ equations of step Daub $_{3}$, resulting in a non-linear system of $N$ equations in $N$ unknowns. Then, solve the system using any iterative numerical procedure, such as Gauss-Siedel, Jacobi or Newton's methods [20], to obtain the high-pass filter $g[\cdot]$.

- STEP Daub ${ }_{5}$ : Obtain the filter $h[\cdot]$ so that $h_{k}=(-1)^{k+1} g_{N-k-1}$ in order to complete the analysis filter pair. If the inverse DWT (IDWT) is required, obtain the filters $\bar{h}[\cdot]$ and $\bar{g}[\cdot]$, so that $\bar{h}_{k}=$ $h_{N-k-1}$ and $\bar{g}_{k}=(-1)^{k+1} h_{k}$, characterizing the re-synthesis filter pair. Lastly, in order to discover the shapes of orthonormal basis associated with the analysis filter pair, as explained in [21], obtain the scaling function $\Phi(x)=\sum_{k} h_{k} \Phi(2 N-k)$ and the wavelet function $\Psi(x)=\sum_{k} g_{k} \Phi(2 N-k)$.

\section{The DST-II}

\subsection{DST-II definition and formulation}

The components of the DST and the DST-II are just the same, i.e., $p[\cdot], q[\cdot], \bar{p}[\cdot], \bar{q}[\cdot], \Gamma(x)$ and $\Theta(x)$. The easiest way to calculate them is to obtain, first, the analysis filter $q[\cdot]$ for which a few restrictions apply regarding the DST-II:

1. the filter support-size is $N \geq 6$. This is required due to the fact that the DST-II has $\frac{N}{2}-2$ vanishing moments in its minor shapelet, equivalently to the wavelet function, as detailed ahead. Therefore, $N<6$ would produce no vanishing moment in such function, disturbing the proposed transform;

2. the filter support-size is necessarily even, as in the DWT theory [3], otherwise a perfect-reconstruction can not be achieved;

3. the signal to be matched, $m[\cdot]$, representing the pattern to be identified by the DST-II, has necessarily an odd size equal to $N+1$.

The particular procedure to determine $q[\cdot]$ is:

- STEPShp ${ }_{1}$ : Force the filter to have unitary energy so that the DST-II preserves that of the input signal, i.e.,

$\sum_{k=0}^{N-1} q_{k}^{2}=1$.

- STEPShp 2 : Impose $\frac{N}{2}-2$ vanishing moments for the major shapelet function, i.e.,

$\sum_{k=0}^{N-1} q_{k} k^{b}=0$,

for $b=0,1, \ldots, \frac{N}{2}-3$; 
- STEPShp ${ }_{3}$ : Define $\frac{N}{2}-1$ orthogonality conditions as in the Daubechies' transform:

$\sum_{k=0}^{N-1} q_{k} q_{k+2 l}=\delta_{0, l}$

- STEPShp 4 : Characterize both the required matching conditions, i.e., $\mathrm{MC}_{1}$ and $\mathrm{MC}_{2}$ :

$\sum_{k=0}^{N-1} q_{k} m_{k}=0$

and

$\sum_{k=0}^{N-1} q_{k} m_{k+1}=0$

- STEPShp $p_{5}$ : Group together the only equation of step $\mathrm{Shp}_{1}$, the

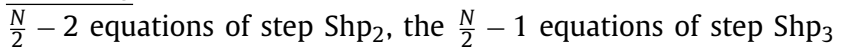
and both matching equations of $\mathrm{Shp}_{4}$, resulting in a non-linear system of $N$ equations in $N$ unknowns. Then, solve the system using any iterative numerical procedure as to obtain the highpass filter $q[\cdot]$.

- STEPShp $\mathrm{p}_{6}$ : Obtain the filter $p[\cdot]$ so that $p_{k}=(-1)^{k+1} q_{N-k-1}$ in order to complete the analysis filter pair. If the inverse DST-II (IDST-II) is required, obtain the filter $\bar{p}[\cdot]$ so that $\bar{p}_{k}=$ $p_{N-k-1}$ and the filter $\bar{q}[\cdot]$ so that $\bar{q}_{k}=(-1)^{k+1} q_{k}$, characterizing the re-synthesis filter pair. Lastly, in order to learn the shapes of orthonormal basis associated with the analysis filter pair, as explained in [14], obtain the major shapelet function $\Gamma(x)=\sum_{k} p_{k} \Gamma(2 N-k)$ and the minor shapelet function $\Theta(x)=\sum_{k} q_{k} \Gamma(2 N-k)$.

Summarizing, the DST-II corresponds to the Daubechies' transform modified so that the two highest vanishing moments are replaced by the matching conditions. $\mathrm{MC}_{1}$ and $\mathrm{MC}_{2}$ are defined in such a way that the scalar products inherent from the DSTII calculation, based on Mallat's algorithm, capture the searched pattern $\left\{m_{0}, \ldots, m_{N}\right\}$ by either matching $\left\{m_{0}, \ldots, m_{N-1}\right\}$ with $\left\{q_{0}, \ldots, q_{N-1}\right\}$ or $\left\{m_{1}, \ldots, m_{N}\right\}$ with $\left\{q_{0}, \ldots, q_{N-1}\right\}$, respectively, depending on the position of $m[\cdot]$ within the input signal being analyzed.

As mentioned above, the procedures used to transform an input signal $s[\cdot]$ to its DST-II and to invert the transformation are entirely based on Mallat's algorithm. They are exactly those described in [14] for the DST and in [18] for the ordinary DWT. Notably, time complexity for both DST-II and DWT is the same.

\subsection{Shape matching and time-frequency analysis}

For shape matching, the DST-II requires a much simpler process in comparison to its predecessor: the more the ith sample of its second-rated part is close to the amplitude 0 , the more the corresponding segment of the original signal under analysis matches $m[\cdot]$. A useful normalized and experimental measure of similarity, emphasizing the presence of zeros in the DST-II $(s[\cdot])$ turning them into the unity, is $\mathbb{S}=e^{-\left(\left|\mathrm{DST}-\mathrm{II}_{1}(s[\cdot])\right|\right)^{\alpha}}$, being $(0<\alpha \leq 1)$. The closer $\mathbb{S}$ is from 0 or 1 , respectively, the less or the more the corresponding segment of the signal under analysis matches $m[\cdot]$.

Specifically, for the $j$ th level DST-II $(s[\cdot])$, the result $\mathbb{S}_{k}=1$ for $k=0,1,2, \ldots$ and $k$ within the second-rated sub-band being examined, implies that the corresponding pattern $m[\cdot]$ starts either at $s_{\left(k \cdot 2^{j}-1\right)}$ or $s_{\left(k \cdot 2^{j}\right)}$, with an extension of $N$ points. Attention is required to the fact that searching for zeros in the $\operatorname{DST}(s[\cdot])$, or equivalently units in $\mathbb{S}$, implies in the inspection of the secondrated part of the transformed signal only, not the master.

For time-frequency analysis, the procedure adopted with the DST-II is exactly the same used for DWTs and DSTs. It is widely

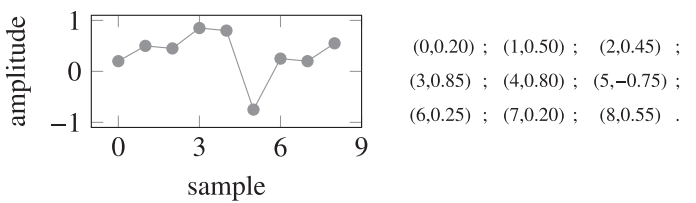

Fig. 1. The example matching signal $m[\cdot]$.

known in the related literature and also explained in [19]. This is the reason why DST-II-based time-frequency analysis is not detailed in this paper.

\section{Numerical example: designing a DST-II}

In this section, a DST-II with support-size $N=8$ is designed to match the signal $m[\cdot]$ that is shown in Fig. 1 together with the numerical values (sample, amplitude) for each one of its $N+1=$ $8+1=9$ points.

According to the algorithm presented in Section 3, the following non-linear system of four groups of equations is required:

- FIRST group: one equation from step $\mathrm{Shp}_{1}$, i.e., $\overline{\sum_{k=0}^{7}\left(q_{k}\right)^{2}}=1$ (from equation 4 ).

- SECOND group: two equations from step $\mathrm{Shp}_{2}$, i.e.,

$$
\left\{\begin{array}{l}
\sum_{k=0}^{7} q_{k}=0 \\
\sum_{k=0}^{7} k q_{k}=0
\end{array} \quad \text { (from equation } 5\right) .
$$

- THIRD group: three equations from step $\mathrm{Shp}_{3}$, i.e.,

$$
\left\{\begin{array}{l}
\sum_{k=0}^{5} q_{k} q_{k+2}=0 \\
\sum_{k=0}^{3} q_{k} q_{k+4}=0 \\
\sum_{k=0}^{1} q_{k} q_{k+6}=0
\end{array}\right.
$$

- FOURTH group: two equations from step $\mathrm{Shp}_{4}$, i.e.,

$$
\left\{\begin{array}{l}
\sum_{k=0}^{7} q_{k} m_{k}=0 \\
\sum_{k=0}^{7} q_{k} m_{k+1}=0
\end{array} \quad\right. \text { (from equations 7and8). }
$$

The non-linear system with eight equations in eight unknowns has the following solution:

$$
\begin{aligned}
q[\cdot]= & \{-0.0834,0.1505,0.5719,-0.7055,-0.0091, \\
& -0.2784,0.2277,0.1263\} .
\end{aligned}
$$

Equivalently,

$$
\begin{aligned}
p[\cdot]= & \{-0.1263,0.2277,0.2784,-0.0091,0.7055,0.5719, \\
& -0.1505,-0.0834\} \\
\bar{p}[\cdot]= & \{-0.0834,-0.1505,0.5719,0.7055 \\
& -0.0091,0.2784,0.2277,-0.1263\} \\
\bar{q}[\cdot]= & \{0.0834,0.1505,-0.5719,-0.7055,0.0091,-0.2784, \\
& -0.2277,0.1263\}
\end{aligned}
$$

In order to find $\Gamma(x)$ and $\Theta(x)$ by using the filters coefficients, the normalization $\sum_{k=0}^{N-1} p_{k}=2$ is first required, since the area under the major shapelet is unitary as in the DWT. Proceeding this 
way, we note that the major shapelet, recursively defined using the dilation equation $\Gamma(x)=\sum_{k} p_{k} \Gamma(2 N-k)$ for a system of support $N=8$, does not exist outside the interval [0-7]. We therefore get:

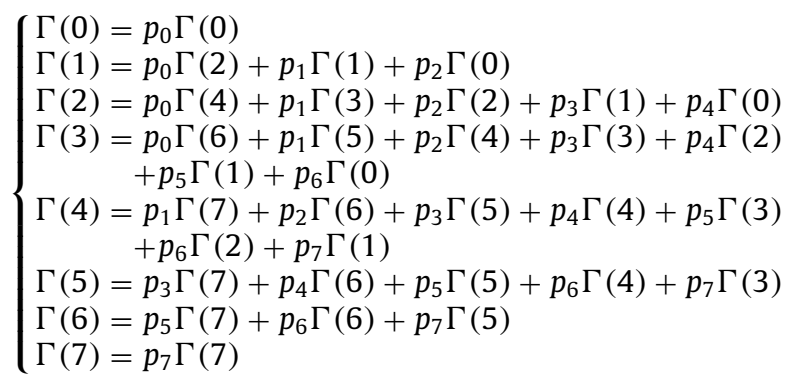

or $M T=T$, being

$M=\left(\begin{array}{cccccccc}p_{0} & 0 & 0 & 0 & 0 & 0 & 0 & 0 \\ p_{2} & p_{1} & p_{0} & 0 & 0 & 0 & 0 & 0 \\ p_{4} & p_{3} & p_{2} & p_{1} & p_{0} & 0 & 0 & 0 \\ p_{6} & p_{5} & p_{4} & p_{3} & p_{2} & p_{1} & p_{0} & 0 \\ 0 & p_{7} & p_{6} & p_{5} & p_{4} & p_{3} & p_{2} & p_{1} \\ 0 & 0 & 0 & p_{7} & p_{6} & p_{5} & p_{4} & p_{3} \\ 0 & 0 & 0 & 0 & 0 & p_{7} & p_{6} & p_{5} \\ 0 & 0 & 0 & 0 & 0 & 0 & 0 & p_{7}\end{array}\right)$ and $T=\left(\begin{array}{c}\Gamma(0) \\ \Gamma(1) \\ \Gamma(2) \\ \Gamma(3) \\ \Gamma(4) \\ \Gamma(5) \\ \Gamma(6) \\ \Gamma(7)\end{array}\right)$.

Thus, matrix $\mathrm{T}$ with scaling function values is the eigenvector of $M$ corresponding to eigenvalue 1 . Using the normalizing condition $\sum_{k} \Gamma(k)=1$, we get:

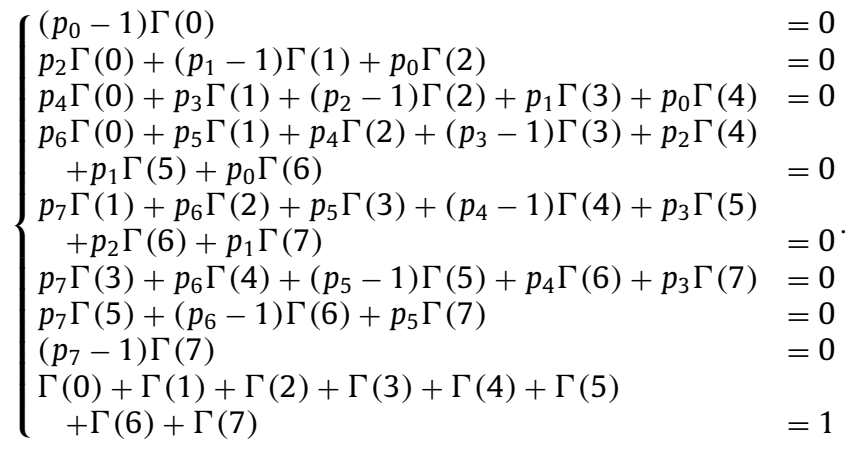

Since $p_{0}, \ldots, p_{7} \neq 0$,

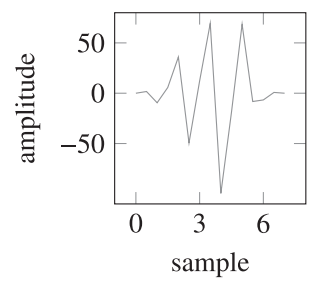

(a) major shapelet

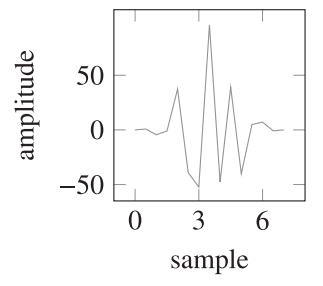

(b) minor shapelet

Fig. 2. The major shapelet and minor shapelet functions, without normalization.

$$
\left\{\begin{aligned}
\Gamma\left(\frac{1}{2}\right)= & p_{0} \Gamma(1)+p_{1} \Gamma(0) \\
\Gamma\left(\frac{3}{2}\right)= & p_{0} \Gamma(3)+p_{1} \Gamma(2)+p_{2} \Gamma(1)+p_{3} \Gamma(0) \\
\Gamma\left(\frac{5}{2}\right)= & p_{0} \Gamma(5)+p_{1} \Gamma(4)+p_{2} \Gamma(3)+p_{3} \Gamma(2) \\
& +p_{4} \Gamma(1)+p_{5} \Gamma(0) \\
\Gamma\left(\frac{7}{2}\right)= & p_{0} \Gamma(7)+p_{1} \Gamma(6)+p_{2} \Gamma(5)+p_{3} \Gamma(4) \\
& +p_{4} \Gamma(3)+p_{5} \Gamma(2)+p_{6} \Gamma(1)+p_{7} \Gamma(0) \\
\Gamma\left(\frac{9}{2}\right)= & p_{2} \Gamma(7)+p_{3} \Gamma(6)+p_{4} \Gamma(5)+p_{5} \Gamma(4)+p_{6} \Gamma(3) \\
& +p_{7} \Gamma(2) \\
\Gamma\left(\frac{11}{2}\right)= & p_{4} \Gamma(7)+p_{5} \Gamma(6)+p_{6} \Gamma(5)+p_{7} \Gamma(4) \\
\Gamma\left(\frac{13}{2}\right)= & p_{6} \Gamma(7)+p_{7} \Gamma(6)
\end{aligned}\right.
$$

Therefore,

$$
\begin{aligned}
&\{\Gamma(0), \Gamma(1 / 2), \Gamma(1), \ldots, \Gamma(13 / 2), \Gamma(7)\} \\
&=\{0,1.694,-9.483,5.68,35.987,-49.438,12.157,69.378, \\
&-99.513,-18.872,68.523,-8.236,-6.670,0.787,0\} .
\end{aligned}
$$

Regarding the minor shapelet, the use of equation $\Theta(x)=$ $\sum_{k} q_{k} \Gamma(2 n-k)$ implies in $\Theta(x)=Q T$, with

$$
Q=\left(\begin{array}{cccccccc}
q_{0} & 0 & 0 & 0 & 0 & 0 & 0 & 0 \\
q_{2} & q_{1} & q_{0} & 0 & 0 & 0 & 0 & 0 \\
q_{4} & q_{3} & q_{2} & q_{1} & q_{0} & 0 & 0 & 0 \\
q_{6} & q_{5} & q_{4} & q_{3} & q_{2} & q_{1} & q_{0} & 0 \\
0 & q_{7} & q_{6} & q_{5} & q_{4} & q_{3} & q_{2} & q_{1} \\
0 & 0 & 0 & q_{7} & q_{6} & q_{5} & q_{4} & q_{3} \\
0 & 0 & 0 & 0 & 0 & q_{7} & q_{6} & q_{5} \\
0 & 0 & 0 & 0 & 0 & 0 & 0 & q_{7}
\end{array}\right)
$$

For the intermediary points, $\Theta\left(\frac{x}{2}\right)=\sum_{k} q_{k} \Gamma(x-k)$. When adopting the same procedure used above for the major shapelet function, the complete result is

$$
\begin{aligned}
\{\Theta(0), \Theta(1 / 2), \Theta(1), \ldots, \Theta(13 / 2), \Theta(7)\} & \\
= & \{0,0.791,-4.432,-1.020,37.413,-39.054,-52.307,96.104, \\
& -47.637,39.105,-40.152,4.896,7.136,-0.842,0\} .
\end{aligned}
$$

Fig. 2 shows the major and minor shapelet functions, without normalization.

\section{Example applications}

5.1. The general prototype to use the DST-II, exemplified for simulated data

This experiment, capable to be used as a prototype to find a pattern within a digital signal, takes advantage of the matching signal $m[\cdot]$, defined in the previous section, and its corresponding DST-II. Particularly, $m[\cdot]$ was embedded within the 64-sample long signal $s[\cdot]$ shown in Fig. 3, so that:

- $s_{i}=\cos \left(\frac{27 \pi i}{8}\right) \cdot \sin \left(\frac{75 \pi i}{8}\right), \quad(0 \leqslant i \leqslant 40)$;

- $s_{i}=m_{i}, \quad(41 \leqslant i \leqslant 49)$;

- $s_{i}=\cos \left(\frac{295 \pi i}{32}\right) \cdot \sin \left(\frac{105 \pi i}{32}\right), \quad(50 \leqslant i \leqslant 63)$.

Later, the algorithm presented in Section 3.2 was applied to $s[\cdot]$, using the first-level DST-II, resulting in the curves shown in Fig. 4:

ger points, whereas the intermediate points satisfy $\Gamma\left(\frac{x}{2}\right)=\sum_{k} p_{k} \Gamma$ $(x-k)$. Thus: 


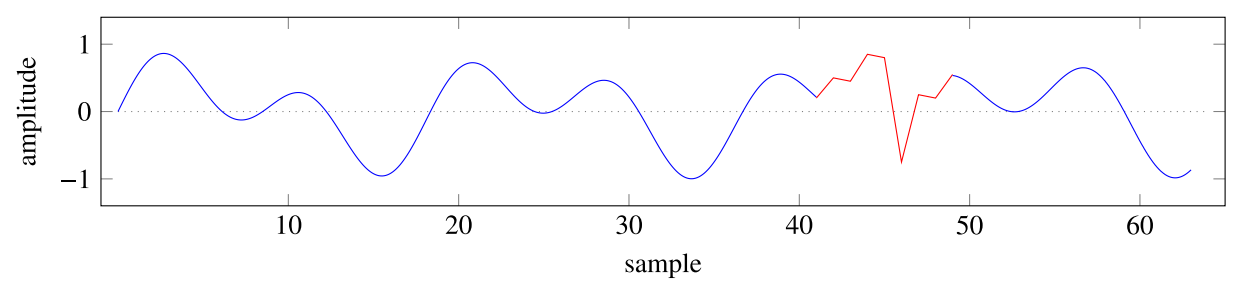

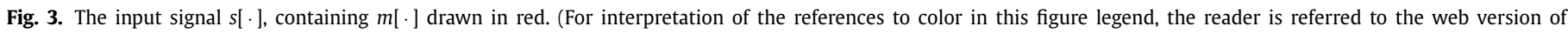
this article.)

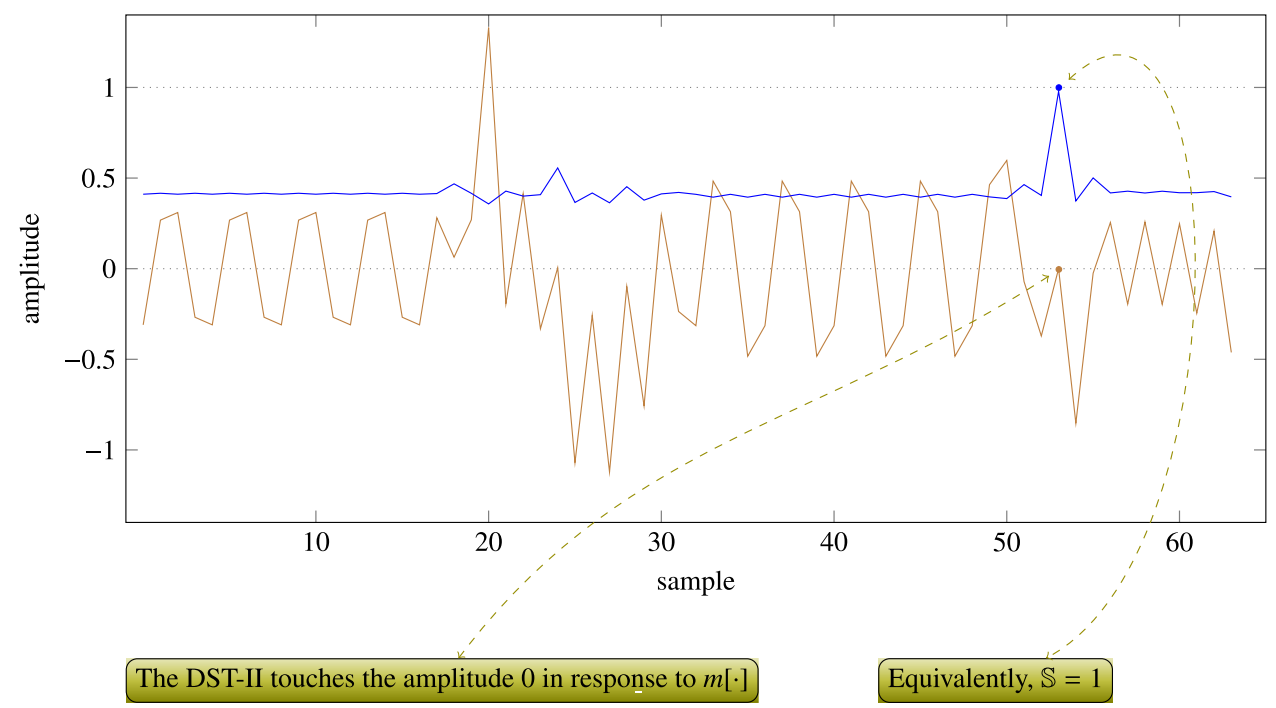

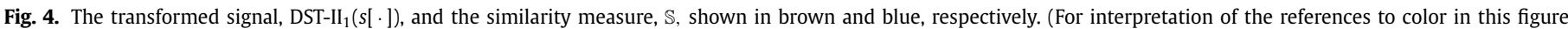
legend, the reader is referred to the web version of this article.)

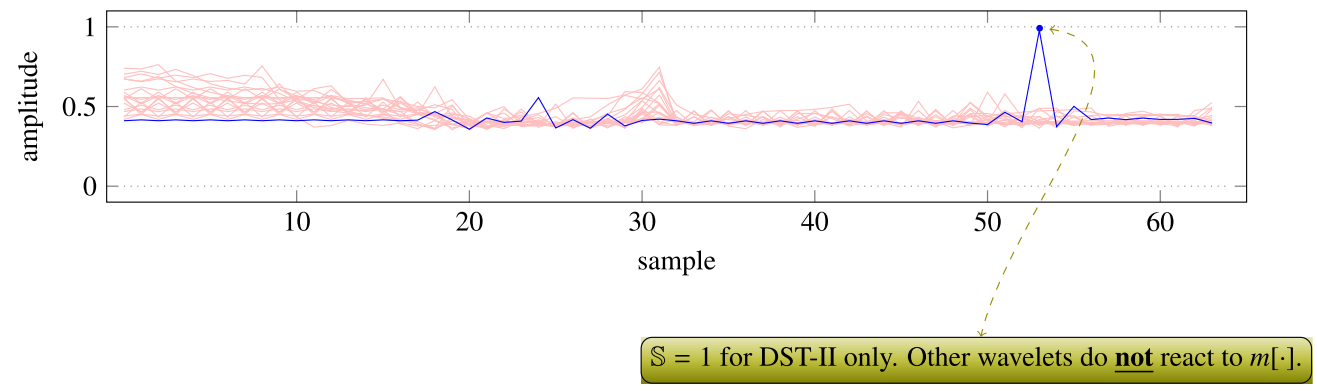

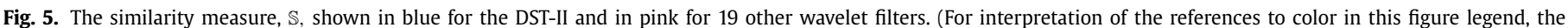
reader is referred to the web version of this article.)

$\operatorname{DST}_{-I_{1}}(s[\cdot])$ and $\mathbb{S}\left(\operatorname{DST}_{-1} \mathrm{II}_{1}(s[\cdot])\right)$ using $\alpha=0.1$, drawn in brown and blue, respectively. In addition to the regular time-frequency information contained in the former, due to the fact that the DSTII is an ordinary wavelet transform, the presence of $m[\cdot]$ within $s[\cdot]$ is confirmed by the corresponding amplitude 1 at the sample 53 of the latter. As explained in Section 3.2, this sample of the transformed signal corresponds to the index $k=21$ of the subband analyzed because, for a first-level DST-II of a 64-sample long signal, the second-rated part starts at index 32, which corresponds to index 0 of that sub-band. Thus, $m[\cdot]$ starts either at $s_{\left(k \cdot 2^{j}-1\right)}=$ $s_{\left(21 \cdot 2^{1}-1\right)}=s_{41}$ or $s_{\left(k \cdot 2^{j}\right)}=s_{\left(21 \cdot 2^{1}\right)}=s_{42}$. As previously defined, we note that $m[\cdot]$ actually starts at $s_{41}$, confirming the proposed theory.

In order to establish a comparison, Fig. 5 shows twenty curves divided into two groups: the blue plot and the nineteen pink plots. Particularly, the former represents $\mathbb{S}\left(\mathrm{DST}_{-} \mathrm{II}_{1}(s[\cdot])\right)$, previously shown in Fig. 4 , and the latter, for which there is no need to distinguish among the curves, correspond to $\mathbb{S}$ applied to each one of the following ordinary wavelet families: Haar, Daubechies with support-sizes 4, 6, 8, 10, 20, 30, 40, 50, 60 and 70, Symmlet with support-sizes 8 and 16 , Coiflets with support-sizes 6,12 , 24 and 30, Beylkin with support-size 18 and, lastly, Vaidyanathan with support-size 24 . Clearly, only the DST-II was capable of identifying $m[\cdot]$ inside $s[\cdot]$. This is expected as only this transform was optimized to match $m[\cdot]$. Certainly, the DST-II always identifies the pattern regardless of its amplitude normalization due to the vanishing Eqs. (7) and (8) specified in Section 3.

\subsection{Analysis of waveform shapes from biological data: spike and overlap sorting}

A simple search on the Web of Science webpage returns thousands of pieces of work related to waveform shape matching. Just to mention a few, [22-27] describe modern approaches which al- 

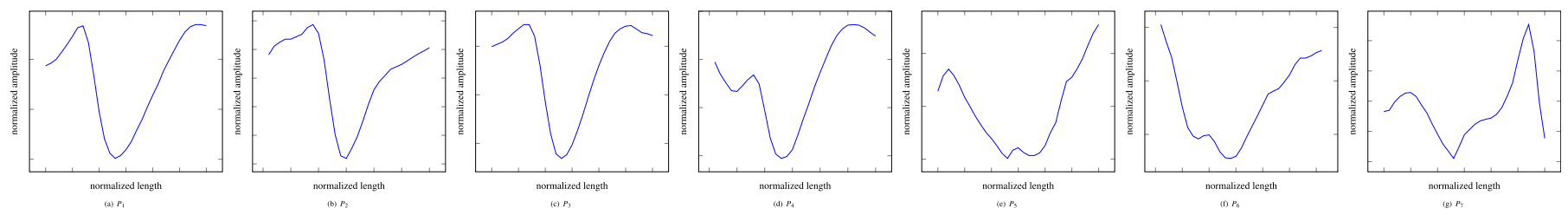

Fig. 6. The 7 patterns used during the tests: $P_{1}, \ldots, P_{7}$.

Table 1

Results for the analyses of $P_{1}, \ldots, P_{7}$ based on DST-II with $\alpha=0.1$. The abbreviation "N.R." means "no reaction".

\begin{tabular}{|c|c|c|c|c|c|c|c|}
\hline - & $P_{1}$ & $P_{2}$ & $P_{3}$ & $P_{4}$ & $P_{5}$ & $P_{6}$ & $P_{7}$ \\
\hline $\mathbb{S}\left(\mathbf{D S T}_{P 1}(\cdot)\right)$ & 1.0 & N.R. & N.R. & N.R. & N.R. & N.R. & N.R. \\
\hline $\mathbb{S}\left(\right.$ DST $\left._{P 2}(\cdot)\right)$ & N.R. & 1.0 & N.R. & N.R. & N.R. & N.R. & N.R. \\
\hline $\mathbb{S}\left(\mathbf{D S T}_{P 3}(\cdot)\right)$ & N.R. & N.R. & 1.0 & N.R. & N.R. & N.R. & N.R. \\
\hline $\mathbb{S}\left(\mathbf{D S T}_{P 4}(\cdot)\right)$ & N.R. & N.R. & N.R. & 1.0 & N.R. & N.R. & N.R. \\
\hline $\mathbb{S}\left(\mathbf{D S T}_{P 5}(\cdot)\right)$ & N.R. & N.R. & N.R. & N.R. & 1.0 & N.R. & N.R. \\
\hline $\mathbb{S}\left(\mathbf{D S T}_{P 6}(\cdot)\right)$ & N.R. & N.R. & N.R. & N.R. & N.R. & 1.0 & N.R. \\
\hline $\mathbb{S}\left(\mathbf{D S T}_{P 7}(\cdot)\right)$ & N.R. & N.R. & N.R. & N.R. & N.R. & N.R. & 1.0 \\
\hline
\end{tabular}

low for the detection of pathologies in a subject's larynx noninvasively. Authors of the papers [28-30] interpret brain activities based on electroencephalogram (EEG) analysis. In paper [31], the authors characterize patterns of $\mathrm{H} 1$, the motion-sensitive neuron of the fly's visual system, by means of their waveform shapes. Similar experiments were also carried out in paper [32]. Thus, this topic is of fundamental importance, as recently confirmed in [33].

In this spike sorting experiment, which is also inspired in those documented in [31], the procedure for pattern classification of biological data extracted from $\mathrm{H} 1$ is repeated using the DST-II. The seven signals shown in Fig. 6, the same presented in [14]-pp.2896 and named $\left\{P_{1}, \ldots, P_{7}\right\}$, were used in such a way that one DSTII was designed for each $N=31$-sample long signal, i.e., DST-II ${ }_{\mathrm{P} 1}$, DST-II $_{\mathrm{P} 2}, \ldots$, DST-II 7 . Then, $7 \cdot 7=49$ all-against-all tests were performed, so that the input $P_{i}$ was compared to the set of signals $\left\{P_{1}, \ldots, P_{7}\right\}$ by using the corresponding DST-II $\mathrm{Pi}_{\mathrm{Pi}}$ in the first level of decomposition. The results obtained when the second-rated part of the first-level transformed inputs were analyzed are shown in Table 1, confirming the efficacy of the procedure. Except for the seven exact matches, for which $\mathbb{S}=1$, we note that there is no reaction (N.R.) when $P_{i}$ is compared to $P_{j}$ for $i \neq j$. For most of the cases and considering $\alpha=0.1$, the absence of reaction means that $\mathbb{S}<0.6$. When the comparisons involve $P_{1}$ and $P_{3}$, which are perceptibly the most similar among the patterns, $\mathbb{S} \approx 0.65$. Particularly, $\mathbb{S}=1$ whenever the exact pattern is found, regardless of its amplitude normalisation. The algorithm based on the DST-II can also be associated with an inspection of the signal energy [32] in order to restrict the detection to those patterns that are above a specific threshold.

Complementing the above-mentioned tests, a set of DSTs-II was designed and successfully employed to detect all the 6325 spikes and overlaps contained in the long signal acquired from $\mathrm{H} 1$, described in [32]-pp.277-278, with 100\% accuracy. This reassures the usability of the proposed approach.

\section{Conclusions}

This paper introduced the second generation of the Discrete Shapelet Transform, i.e., DST-II, a novel tool for TFS joint analysis of discrete-time signals. A brief literature review followed by the detailed construction of the proposed transform were presented, together with a numerical example, a prototype to search for a generic pattern in a digital signal and an application on spike and overlap sorting.
The new transform extends the traditional DWT decomposition in such a way that a shape analysis becomes possible, improving its predecessor, i.e., the DST. The results obtained for pattern matching and the comparisons with other wavelets reassure the efficacy of the proposed approach, which may be applied to a variety of signals without restrictions. Although the first generation of DSTs can also be used to identify patterns contained in a certain input signal, it requires much more work to build and solve the non-linear system of equations that produces the filter bank, as can be clearly seen in paper [14]. Additionally, except by the first generation of the DST, there is no other tool designed for TFS joint analysis. Thus, the contribution of this work for the field of information fusion is clear.

On one hand, the DST-II presents the following advantages: (i) it is built to match a pattern within a digital signal, regardless of its amplitude normalization, (ii) it does not require iterative training procedures to learn the pattern as a neural network or a deep learning algorithm [34] does, (iii) it is easily computed and presents the same time complexity of any DWT with the corresponding support-size. On the other hand, the main weakness is that the DST-II is capable to match one pre-defined pattern, but not a set as a statistical learning algorithm does. Furthermore, its robustness against noise is modest. One special remark is that, whenever a DWT is required for some reason, it can be replaced by the DST, thus allowing not only a time-frequency but a TFS joint analysis. Furthermore, both a wavelet analysis and a waveletpacket analysis [3] can be performed based on the DST-II.

An important note: for pattern matching and shape analysis, the IDST-II is not required, however, depending on the specific application, for instance, TFS analysis and compression, where some coefficients of the transformed signal are modified prior to reconstruction, the inverse is needed. This justifies the design of orthogonal PRFBs and the specification of $\Gamma(x)$ and $\Theta(x)$.

For researchers interested in the proposed approach, $\mathrm{C} / \mathrm{C}++$ [35] source code to design a DST-II from an arbitrary matching signal $m[\cdot]$ is available upon prior request ${ }^{1}$.

\section{Acknowledgments}

I am very grateful to CNPq - "Conselho Nacional de Desenvolvimento Científico e Tecnológico", in Brazil, for the grants provided, through process number 306811/2014-6, to support this research.

\section{References}

[1] A. Haar, Zur theorie der orthogonalen funktionensysteme, (Erste Mitteilung). Math. Ann. (1910) 331-371. - PhD thesis.

[2] I. Daubechies, Ten Lectures on Wavelets, Society for Industrial and Applied Mathematics, Philadelphia-PA, 1992.

[3] G. Strang, T. Nguyen, Wavelets and Filter Banks, Wellesley-Cambridge Press, Wellesley, 1997.

[4] S. Li, X. Kang, L. Fang, J. Hu, H. Yin, Pixel-level image fusion: a survey of the state of the art, Inf. Fusion 33 (January) (2017) 100-112.

[5] H. Ghassemian, A review of remote sensing image fusion methods, Inf. Fusion 32-A (November) (2016) 75-89.

[6] Y. Liu, S. Liu, Z. Wang, A general framework for image fusion based on multi-scale transform and sparse representation, Inf. Fusion 24 (July) (2015) 147-164.

\footnotetext{
${ }^{1}$ Please, send requests to guido@ieee.org
} 
[7] Z.-H. Huang, W.J. Li, J. Wang, T. Zhang, Face recognition based on pixel-level and feature-level fusion of the top-levels wavelet sub-bands, Inf. Fusion 22 (March) (2015) 95-104.

[8] R. Singh, A. Khare, Fusion of multimodal medical images using daubechies complex wavelet transform - a multiresolution approach, Inf. Fusion 19 (September) (2014) 49-60.

[9] Y. Shi, X. Yang, T. Cheng, Pansharpening of multispectral images using the nonseparable framelet lifting transform with high vanishing moments, Inf. Fusion 20 (November) (2014) 213-224.

[10] S. Li, B. Yang, J. Hu, Performance comparison of different multi-resolution transforms for image fusion, Inf. Fusion 12 (n.2) (2011) 74-84.

[11] S. Yang, M. Wang, L. Jiao, R. Wu, Z. Wang, Image fusion based on a new contourlet packet, Inf. Fusion 11 (n.2) (Apr-2010) 78-84.

[12] Y. Zheng, E.A. Essock, B.C. Hansen, A.M. Haun, A new metric based on extended spatial frequency and its application to DWT based fusion algorithms, Inf. Fusion 8 (n.2) (2007) 177-192.

[13] J.J. Lewis, R.J. O'Callaghan, S.G. Nikolov, D.R. Bull, N. Canagarajah, Pixel- and region-based image fusion with complex wavelets, Inf. Fusion 8 (n.2) (2007) $119-130$.

[14] R.C. Guido, S. Barbon Jr, L.S. Vieira, et al., Introduction to the discrete shapelet transform and a new paradigm: joint time-frequency-shape analysis, in: Proc. IEEE International Symposium on Circuits and Systems (IEEE ISCAS 2008), Seattle, WA, USA, vol. 1, 2008, pp. 2893-2896.

[15] R.C. Guido, p. Addison, J. Walker, Introducing wavelets and time-frequency analysis, IEEE Eng. Biol. Med. Mag. 28 (n.5) (2009) 13.

[16] K. Falconer, Fractal Geometry: Mathematical Foundations and Applications, 3.ed., Wiley, 2014

[17] A.V. Oppenheim, R.W. Schafer, Discrete-time Signal Processing, 3.ed., Prentice-Hall, 2009.

[18] R.C. Guido, Practical and useful tips on discrete wavelet transforms, IEEE Signal Process. Mag. 32 (n. 3) (2015) 162-166.

[19] R.C. Guido, Effectively interpretating discrete wavelet transforms signals, IEEE Signal Process. Mag. 34 (n.3) (2017) 89-100.

[20] R.L. Burden, J.D. Faires, A.M. Burden, Numerical Analysis, 10.ed., Cengage Learning, 2015.

[21] R.C. Guido, A note on a practical relationship between filters coefficients and the scaling and wavelet functions of the discrete wavelet transform, Appl. Math. Lett. 24 (n.7) (2011) 1257-1259.
[22] E. Fonseca, R.C. Guido, P.R. Scalassara, C.D. Maciel, J.C. Pereira, Wavelet time-frequency analysis and least-squares support vector machine for the identification of voice disorders, Comput. Biol. Med. 37 (n. 4) (2007) 571-578.

[23] R.C. Guido, ZCR-aided neurocomputing: a study with applications, Knowl. Based Syst. 105 (2016) 248-269.

[24] D. Panek, A. Skalski, J. Gajda, R. Tadeusiewicz, Acoustic analysis assessment in speech pathology detection, Int. J. Appl. Math. Comput. Sci. 25 (n.3) (2015) 631-643.

[25] M. Alsulaiman, Voice pathology assessment systems for dysphonic patients: detection, classification, and speech recognition, IETE J. Res. 60 (n.2) (2014) $156-167$.

[26] M.J. Pulga, A.C. Spinardi-Panes, S.A. Lopes-Herrera, L.P. Maximino, Evaluating a speech-language pathology technology, Telemed. e-health 20 (n.3) (2014) 269-271.

[27] T.L. Whitehill, S. Bridges, K. Chan, Problem-based learning (PBL) and speech-language pathology: a tutorial, Clin. Linguist. Phon. 28 (n.1-2) (2014) $5-23$.

[28] T.P. Deepa, H.S. Vinay, Classification of motion and speech based brain EEG signals using bilayer bayesian classifier with association rule (BBC-AR), Int. J. Comput. Sci. Mobile Comput. 3 (n.10) (Oct-2014) 777-782.

[29] J.S. Brumberg, et al., Brain-computer interfaces for speech communication, Speech Commun. 52 (n.4) (2010) 367-379.

[30] D.A. Rojas, L.A. Góngora, O.L. Ramos, EEG signal analysis related to speech process through BCI device EMOTIV, FFT and statistical methods, ARPN J. Eng. Appl. Sci. 11 (n.5) (2016) 1-11.

[31] R.C. Guido, J.F.W. Slaets, R. Koberle, L.O.B. Almeida, J.C. Pereira, A new technique to construct a wavelet transform matching a specified signal with applications to digital, real-time, spike and overlap pattern recognition, Digit. Signal Process. 16 (n. 1) (Jan-2006) 24-44.

[32] R.C. Guido, A tutorial on signal energy and its applications, Neurocomputing 179 (Feb-2016) 264-282.

[33] S.R. Cole, Brain oscillations and the importance of waveform shape, Trends Cogn. Sci. 21 (n.2) (Feb-2017) 137-149.

[34] A. Gibson, J. Patterson, Deep Learning: A Practitioner's Approach, O'Reilly Media, 2016.

[35] B. Stroustrup, The C++ Programming Language, 4. ed., Addison-Wesley Professional, 2013. 